\title{
Exploration of the immune-related signature and immune infiltration analysis for breast ductal and lobular carcinoma
}

\author{
Bochuan Zhang ${ }^{1}$, Qingfeng Wang ${ }^{2}$, Chenghao $\mathrm{Fu}^{3}$, Chunying Jiang ${ }^{3}$, Shiliang $\mathrm{Ma}^{1,3}$ \\ ${ }^{1}$ College of Food Science and Technology, Shenyang Agricultural University, Shenyang 110161, China; ${ }^{2}$ Basic Medical College Liaoning \\ University of Traditional Chinese Medicine, Shenyang 110847, China; ${ }^{3}$ College of Bioscience and Biotechnology, Shenyang Agricultural \\ University, Shenyang 110161, China \\ Contributions: (I) Conception and design: B Zhang, C Jiang, S Ma; (II) Administrative support: Q Wang; (III) Provision of study materials or patients: \\ C Fu; (IV) Collection and assembly of data: B Zhang; (V) Data analysis and interpretation: B Zhang, C Fu; (VI) Manuscript writing: All authors; (VII) \\ Final approval of manuscript: All authors. \\ Correspondence to: Shiliang Ma. College of Food Science and Technology, Shenyang Agricultural University, Shenyang 110161, China. Email: msl@syau.edu.cn; \\ Qingfeng Wang. Basic Medical College Liaoning University of Traditional Chinese Medicine, Shenyang 110847, China. Email: Intcmwqf@foxmail.com.
}

Background: In this study, we aimed to explore the tumour associated immune signature of breast cancer (BC) and conduct integrative analyses with immune infiltrates in BC.

Methods: We downloaded the transcriptome profiling and clinical data of BC from The Cancer Genome Atlas (TCGA) database. The list of immune-related signatures was from the Innate database. The limma package was utilized to conduct the normalization, and we screened the differential immune signatures in BC. A univariate Cox regression model and the LASSO method were used to find the hub prognostic immune genes. The TAIG risk model was calculated based on the multivariate Cox regression results, and a receiver operating characteristic (ROC) curve was generated to assess the predictive power of TAIG. Moreover, we also conducted a correlation analysis between TAIG and the clinical characteristics. Additionally, we utilized the METABRIC cohort as the validation data set. The TIMER database is a comprehensive resource for performing systematic analyses of immune infiltrates across various malignancies. We evaluated the associations of immune signatures with several immune cells based on TIMER. Furthermore, we used the CIBERSORT algorithm to determine the fractions of immune cells in each sample and compared the differential distributions of immune infiltrates between two TAIG groups using the Wilcoxon rank-sum test.

Results: A total of 1,178 samples were obtained from the TCGA-BRCA database, but only 1,045 breast tumour samples were matched with complete transcriptome expression data. Meanwhile, we collected a total of 1,094 BC patients from the METABRIC cohort. We found a list of 1,399 differential immune signatures associated with survival, and functional analysis revealed that these genes participated in cytokine-cytokine receptor interactions, Th1 and Th2 cell differentiation and the JAK-STAT signalling pathway. The TAIG risk model was established from the multivariate Cox analysis, and we observed that high TAIG levels correlated with poor survival outcomes based on Kaplan-Meier analysis. The Kruskal-Wallis test suggested that high TAIG levels correlated with high AJCC-TNM stages and advanced pathological stages $(\mathrm{P}<0.01)$. We validated the well robustness of TAIG in METABRIC cohort and 5-year AUC reached up to 0.829. Moreover, we further uncovered the associations of hub immune signatures with immune cells and calculated the immune cell fractions in specific tumour samples based on gene signature expression. Last, we used the Wilcoxon ranksum test to compare the differential immune density in the two groups and found that several immune cells had a significantly lower infiltrating density in the high TAIG groups, including $\mathrm{CD}^{+} \mathrm{T}$ cells $(\mathrm{P}=0.031)$, memory resting $\mathrm{CD}^{+} \mathrm{T}$ cells $(\mathrm{P}=0.026), \mathrm{M} 0$ macrophages $(\mathrm{P}=0.023)$, and $\mathrm{M} 2$ macrophages $(\mathrm{P}=0.048)$.

Conclusions: In summary, we explored the immune signature of BC and constructed a TAIG risk model to predict prognosis. Moreover, we integrated the identified immune signature with tumour-infiltrating immune cells and found adverse associations between the TAIG levels and immune cell infiltrating density. 
Keywords: Tumour-associated immune signature; immune infiltrates; prognosis; breast cancer (BC)

Submitted Jul 25, 2019. Accepted for publication Nov 08, 2019.

doi: 10.21037/atm.2019.11.117

View this article at: http://dx.doi.org/10.21037/atm.2019.11.117

\section{Introduction}

Breast cancer (BC) has gradually become a commonly diagnosed cancer type worldwide. According to Globocan (1), there are 2,088,849 women who are diagnosed with $\mathrm{BC}$ and 626,679 patients who die from it per year; $\mathrm{BC}$ has the highest incidence/mortality among female malignant tumour types. Surgical and drug therapies are considered the most common treatments for BC. However, both methods result in physical injury, coupled with poor prognosis and high recurrence $(2,3)$. With the accumulation of molecular research on $\mathrm{BC}$, targeted therapy has gradually become a key means of treatment (4). Trastuzumab (5), temsirolimus (6) and bevacizumab (7) are common targeted drugs in the clinic. There are abundant clinical studies on differential gene expression in BC cases, which could provide accurate differential gene data. This could supply the theoretical basis for $\mathrm{BC}$ targeted therapy research and unearth novel targets for clinical diagnosis, treatment and prognosis of $\mathrm{BC}$.

The immune response is considered an important pathway in the occurrence and prognosis of $\mathrm{BC}$, which means that the enhancement of the tumour immune response could provide an effective benefit for those with BC, improving clinical results and overall survival (OS) (8). Immunotherapies such as the anti-growth factor 2 (HER2) Th1 response are becoming a favourable treatment approach for BC. For example, by blocking HER2, the Th1 response was inhibited, and $40-67 \%$ of patients achieved a pathologic complete response (pCR) (9). Another study discovered the importance of immune escape in tumour cell chemotherapy resistance, where programmed death-1 (PD-1)/programmed death ligand 1 (PD-L1) inhibitors could be regarded as a potential approach in the triple-negative breast cancer (TNBC) (10). Studies have shown that the components of the tumour immune environment, especially the tumour-infiltrating lymphocytes (TIL) communities, are correlated with patients' disease-free survival (DFS) and OS (11). However, the previous investigations mainly focused on the different gene expression (DGE) or the tumour immune environment of BC. The biomarkers of tumour immune cells for BC are still poorly understood, and feasible immune biomarkers for the prediction of BC patients' prognosis are lacking. Moreover, as Egelston's research has shown (12), although TIL can be regarded as the immune signature of the tumour, the complex function of different cell subsets in TIL limits its utilization in $\mathrm{BC}$ research. Therefore, it is essential to confirm the relationship of specific biomarkers of tumour immune cells and $\mathrm{BC}$ and find a robust immune target that can serve as a perspective of tumour immunology and may become a target for immunotherapy of BC.

In this study, we utilized the transcriptome data from The Cancer Genome Atlas (TCGA) to develop an immunerelated risk signature for $\mathrm{BC}$, and we validated 1,399 immune-related genes which correlated to the OS of BC. To evaluate the clinical value of these signatures, the TAIG risk model was calculated based on the multivariate Cox regression results, and we conducted the correlation analysis between TAIG and the clinical characteristics. To further investigate the molecular and immune profiling of the signature, we evaluated the associations between the immune signature and several immune cells based on TIMER.

\section{Methods}

\section{Data acquisition and processing}

We downloaded the genome expression data of 1,178 samples from the TCGA database (https://portal. gdc.cancer.gov/), including 1,066 tumour samples and 112 matched normal samples. Besides, we collected a total of 1,094 patients from the METABRIC cohort with complete survival information and expression data. Meanwhile, we obtained a list of immune related signatures from the InnateDB database (https://www.innatedb.ca/), which provides available resources for immunology research. Then, limma package was utilized to determine the normalization of gene expression, and we conducted a differential analysis to screen the abnormally expressed genes in tumour versus normal samples. We then obtained the intersect immune signature on a Venn diagram. To investigate the biological function of differential immune genes in BC, we transferred the gene symbol with Entrez 
ID and performed the Gene Ontology (GO) and KEGG analyses via the clusterProfiler, org.Hs.eg.db, enrichplot and ggplot2 packages. The significantly enriched GO items and signalling pathways are shown using bar plots and dot plots. Meanwhile, we also obtained the clinical information of patients from the database via the TCGAbiolinks package, including age, gender, TNM stages, tumour grades, and follow-up with vital status.

\section{Construction of the TAIG risk score in BC}

Since we screened the differentially expressed immune genes, we further investigated the hub immune genes for predicting the prognosis of BC. First, we selected the differential immune genes and conducted the univariate Cox regression analysis to find the prognostic signature among them. Then, we chose the significant signatures with $\mathrm{P}<0.001$ and prepared a Least Absolute Shrinkage And Selection Operator (LASSO) model to reduce the variables. Afterwards, we conducted a multivariate Cox analysis to construct the TAIG (tumor-associated immune genes) risk model calculated as: TAIG $=\Sigma\left(\beta_{\mathrm{i}} \times \operatorname{Exp}_{\mathrm{i}}\right)$, where $\beta_{\mathrm{i}}$, the coefficients, represented the weight of the respective signature and $\operatorname{Exp}_{\mathrm{i}}$ represented the expression value. Accordingly, we could calculate the TAIG risk score for each patient and classified patients into a high- or lowTAIG group with the median value as the cut-off data. The distribution of the vital status was illustrated according to the TAIG risk score levels. We also showed the differentially expressed levels of immune signatures in two TAIG groups via a pheatmap package.

\section{Assessment of TAIG and associations with clinical variables}

Given the identification of hub immune signatures associated with survival outcomes in BC, we intended to explore the predictive value of TAIG. We utilized the timeROC package to conduct the receiver operating characteristic (ROC) curve to show the 5-year OS prediction. Meanwhile, a Kaplan-Meier analysis with a logrank test was used to assess the survival difference between low- and high-TAIG groups. Moreover, we discovered the underlying relationships between TAIG and several clinical features consisting of TNM stages or pathological stages. The Wilcoxon rank-sum test was utilized for comparing differential levels of TAIG between two groups, but the Kruskal-Wallis test was appropriate when dealing with three or more groups.

\section{Explorations of associations between TAIG and immune infiltrates}

TIMER is a comprehensive resource for systematic analysis of immune infiltrates across multiple malignancies. The abundances of six immune infiltrates (B cells, $\mathrm{CD}^{+} \mathrm{T}$ cells, $\mathrm{CD} 8^{+} \mathrm{T}$ cells, neutrophils, macrophages and dendritic cells) are estimated by the statistical method, which is validated by pathological estimations. This web server allows investigators to input function-specific parameters, with resulting figures dynamically displayed to conveniently access the tumour's immunological, clinical, and genomic features. Based on the public resources, we could discover the identified immune signature with tumour-infiltrating immune cells. Pearson's correlation coefficient and the estimated $\mathrm{P}$ value were calculated to evaluate the associations between TAIG and immune infiltration cells.

\section{CIBERSORT and differential abundance of immune cells in two TAIG groups}

Given the important roles of tumour-infiltrating immune cells in the tumour microenvironment, we utilized CIBERSORT, a newly developed deconvolution algorithm, to determine the fractions of 22 immune cells in each sample. We exhibited the results as a box plot, and the various immune cells were annotated under the legend. Furthermore, we conducted a Wilcoxon rank-sum test to compare the differential abundance of immune cells in the two TAIG groups. Last, we illustrated the differential density of immune cells in two TAIG groups using a pheatmap package, where the colours ranging from green to red represented the low to high infiltrating levels.

\section{Statistical analysis}

The Student's $t$-test was suitable for continuous variables, while categorical variables were compared by a Chisquare $\left(\chi^{2}\right)$ test. A Wilcoxon rank-sum test was a nonparametric statistical test mainly utilized for comparing two groups, and a Kruskal-Wallis test was used for two or more groups. Differential analysis and normalization were mainly conducted using the "limma" package. The Cox regression model or Kaplan-Meier analysis with a logrank test was performed using the "survival" package. All statistical analyses were performed using $\mathrm{R}$ software (Version 3.5.2), and a $\mathrm{P}$ value $<0.05$ was considered to be statistically significant. 
Table 1 Baseline characteristics of 1,053 breast cancer patients included in this analysis

\begin{tabular}{|c|c|}
\hline Variables & Count (\%) \\
\hline Age (mean $\pm S D)$ & $58.24 \pm 13.19$ \\
\hline Follow-up (y) & $3.33 \pm 3.22$ \\
\hline \multicolumn{2}{|l|}{ Status } \\
\hline Alive & 915 (86.89) \\
\hline Dead & $138(13.11)$ \\
\hline \multicolumn{2}{|l|}{ Gender } \\
\hline Male & $12(1.14)$ \\
\hline Female & 1,041 (98.86) \\
\hline \multicolumn{2}{|l|}{ AJCC-T } \\
\hline $\mathrm{T} 1$ & $270(25.64)$ \\
\hline $\mathrm{T} 2$ & $614(58.31)$ \\
\hline T3 & $129(12.25)$ \\
\hline T4 & $37(3.51)$ \\
\hline Unknow & $3(0.28)$ \\
\hline \multicolumn{2}{|l|}{ AJCC-N } \\
\hline NO & $484(45.96)$ \\
\hline N1 & 354 (33.62) \\
\hline N2 & $119(11.30)$ \\
\hline N3 & $76(7.22)$ \\
\hline Unknow & $20(1.90)$ \\
\hline \multicolumn{2}{|l|}{ AJCC-M } \\
\hline MO & $876(83.19)$ \\
\hline M1 & $22(2.09)$ \\
\hline Unknow & 155 (14.72) \\
\hline \multicolumn{2}{|l|}{ Pathological stage } \\
\hline I & $172(16.33)$ \\
\hline II & $597(56.70)$ \\
\hline III & $240(22.79)$ \\
\hline IV & $20(1.90)$ \\
\hline Unknow & $24(2.28)$ \\
\hline \multicolumn{2}{|l|}{ Grade } \\
\hline G1 & - \\
\hline G2 & - \\
\hline G3 & - \\
\hline G4 & - \\
\hline Unknow & $1,053[100]$ \\
\hline \multicolumn{2}{|l|}{ TAIG score } \\
\hline Low & $523(49.67)$ \\
\hline High & $522(49.57)$ \\
\hline Unknow & $8(0.76)$ \\
\hline
\end{tabular}

TCGA, The Cancer Genome Atlas; AJCC, American Joint Committee on Cancer; TAIG, Tumor Associated Immune Genes.

\section{Results}

\section{Identification of prognostic immune-related signatures in $B C$}

We obtained 1,178 samples from the TCGA database, consisting of 1,066 tumour samples and 112 corresponding normal samples. We excluded the patients with insufficient clinical data, and the average age was $58.24 \pm 13.19$ among the 1,053 tumour samples. The complete information is shown in Table 1. Meanwhile, the corresponding clinical information of METABRIC patients was collected in http:// cdn.amegroups.cn/static/application/64f39528984716b9083 7edd92e9d54e9/10.21037atm.2019.11.11-1.xlsx. We found 1,045 tumour samples that were matched with complete survival and transcriptome data and were included in our subsequent study. We used the limma package to conduct the normalization of transcriptome profiles and screened approximately 10,563 differentially expressed genes with $\mid \log$ fold changel $>1$ and false discovery rate $(\mathrm{FDR})<0.05$. The volcano plot is shown in Figure 1 to exhibit the upregulated or down-regulated genes. We downloaded a list of 4,678 immune-related genes from the InnateDB database to intersect approximately 1,399 differential immune-related signatures, which is shown in a Venn diagram (Figure 1). The functional enrichment analysis revealed that these differentially expressed immune genes participated in several areas of immune-related crosstalk, including cytokine-cytokine receptor interactions, Th1 and Th2 cell differentiation and the JAK-STAT signalling pathway.

\section{Establishment of TAIG risk score and model assessment}

Given the fact that these immune related signatures showed differential levels in tumour versus normal samples, we further wanted to screen the prognostic genes among them. First, we merged the expression profiles of differential immune genes with survival data and utilized the univariate Cox analysis to obtain a list of 136 prognostic signature with $\mathrm{P}<0.01$. Then, we selected 136 genes with which to perform the LASSO regression method to find the most significantly prognostic immune genes. As the screening procedure shows in Figure 1, we identified the 17 hub immune genes shown in Table 2. Then, we performed a multivariate Cox regression analysis to calculate each coefficient of signature, which represented the weight of each gene. The TAIG risk score was accordingly calculated as: TAIG $=-0.050499 \times$ ADAMTS $8-0.041650 \times$ ADRB $1+0.197286 \times$ APOA5 $-0.074406 \times$ CCL1 -0.109735× CDHR4 -0.074999x FLT3 
A

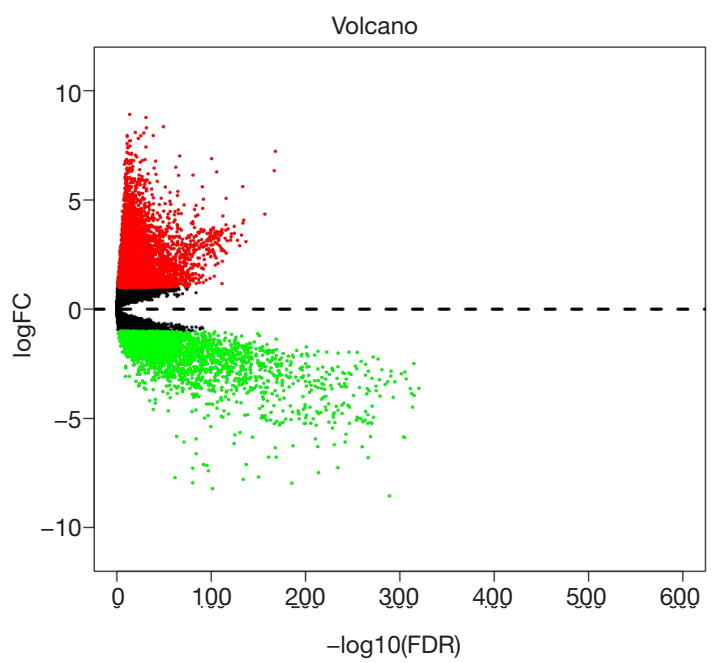

B

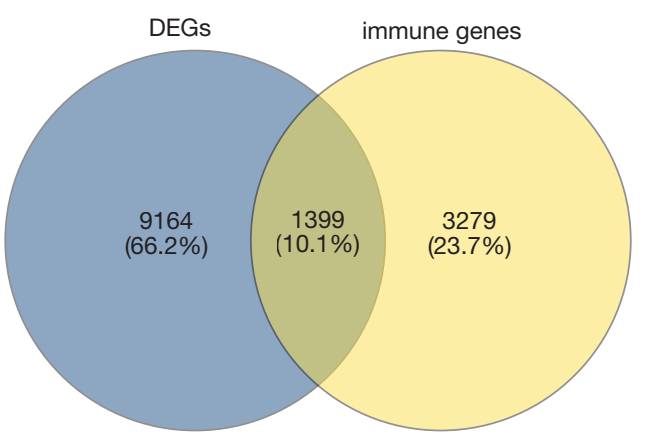

D

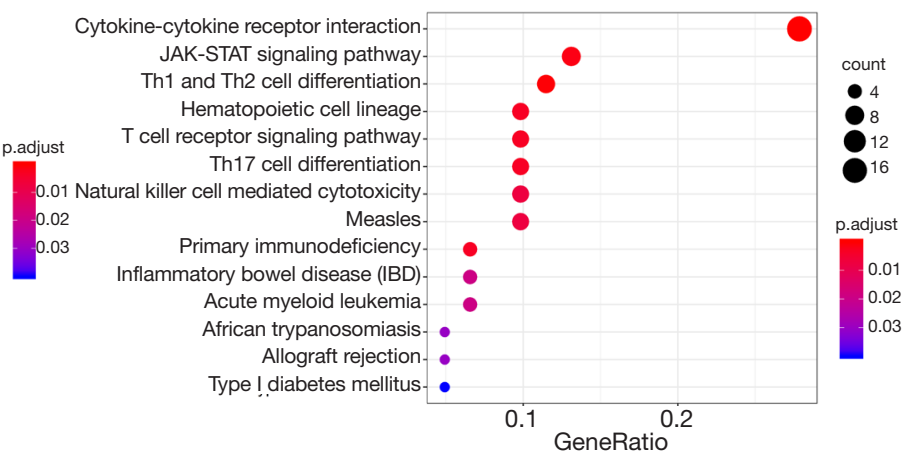

$\mathrm{F}$

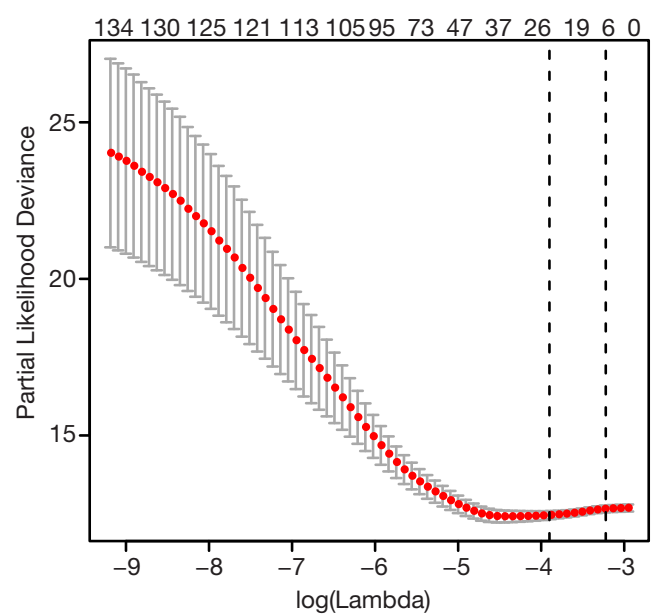

Figure 1 Identification of hub immune signature in breast cancer. (A) Volcano plot was drawn to show the differentially expressed genes in breast cancer versus normal samples; (B) the intersect immune signature was associated with survival from the univariate Cox regression method with $\mathrm{P}<0.01$, where the list of immune signature on the right was obtained from InnateDB database; (C,D) functional enrichment analysis uncovered the potential immune related crosstalk associated with prognostic immune signature; (E,F) LASSO regression method was conducted to search the hub 17 tumor-associated immune signature. 
Table 2 Identification of 17 hub prognostic immune signature based on multivariate regression method

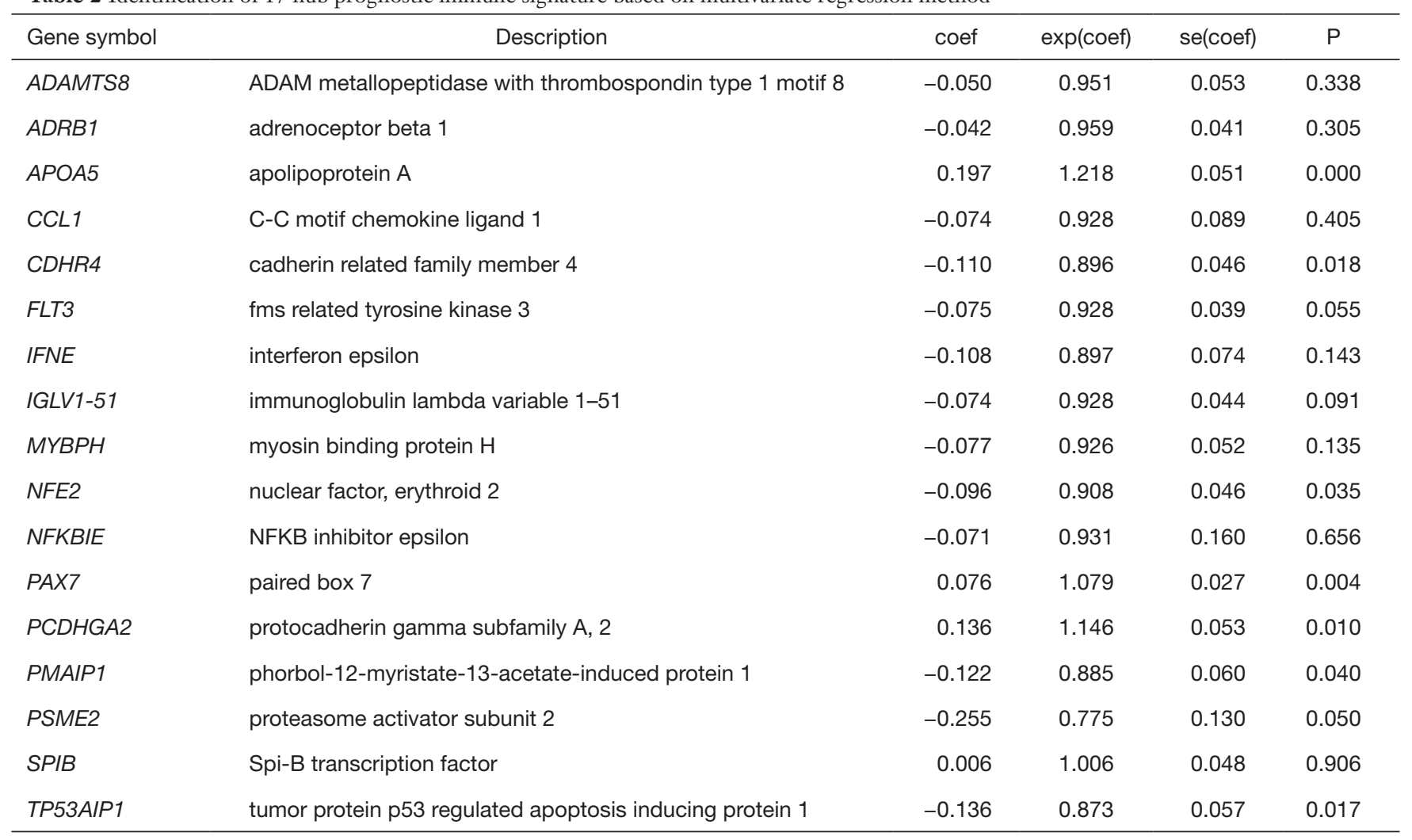

$-0.108292 \times$ IFNE $-0.074270 \times$ IGLV1-51 -0.077379x MYBPH $-0.096452 \times$ NFE2 $-0.071412 \times$ NFKBIE $+0.076329 \times \mathrm{PAX} 7+0.136178 \times$ PCDHGA2 -0.122293x SPIB $-0.135507 \times$ TP53AIP1. We thus could classify the 1045 BC patients into 523 low-TAIG and 522 high-TAIG groups. We observed that the patients with a high TAIG risk score suffered more survival risks, as shown in Figure 2. Moreover, the differentially expressed levels of hub genes in the two TAIG groups are shown by the heatmap in Figure 2. What is more, the AUC of the ROC plot in Figure 3 was 0.793 , indicating the superior predictive accuracy of the TAIG risk score for the prognosis of BC. The Kaplan-Meier analysis with the log-rank test also further demonstrated that patients with high TAIG scores were associated with poor $\mathrm{OS}(\mathrm{P}<0.001)$ in Figure 3. In addition, the correlation analysis between TAIG levels and clinical characteristics also revealed that high TAIG levels correlated with high AJCC-TNM stages and advanced pathological stages $(\mathrm{P}<0.01)$, implicating the significant clinical value of TAIG. Last, we further validated the TAIG in an independent data set in METABRIC cohort and found that the 3-, 5- and 7-year AUC in predicting OS was 0.732,
0.784 and 0.829 , respectively (Figure 4). Correspondingly, the Kaplan-Meier curve in 1,094 patients showed that the higher TAIG risk scores indicated poor survival outcomes with $\mathrm{P}<0.0001$ (Figure 4).

\section{Associations of TAIG with tumour-infiltrating immune cells}

Given the important roles of infiltrating immune cells in the tumour microenvironment, we integrated the comprehensive analysis of immune signatures combined with immune infiltrates. Based on the TIMER database, we discovered the relationships between 17 hub immune signatures and tumour purity or several important immune cells. We observed that most of these signatures were associated with immune cells, especially PCDHGA2, SPIB, ADRB1, FLT3, and NFKBIE, which are shown in Figure 5.

Moreover, we further utilized the CIBERSORT algorithm to determine the estimated fractions of 22 immune cells in each sample. We excluded the samples with a calculated $\mathrm{P}$ value $>0.05$ to guarantee the accuracy of the analysis, and we illustrated the results in a box plot, where the differential immune cells were annotated with various colours and the 

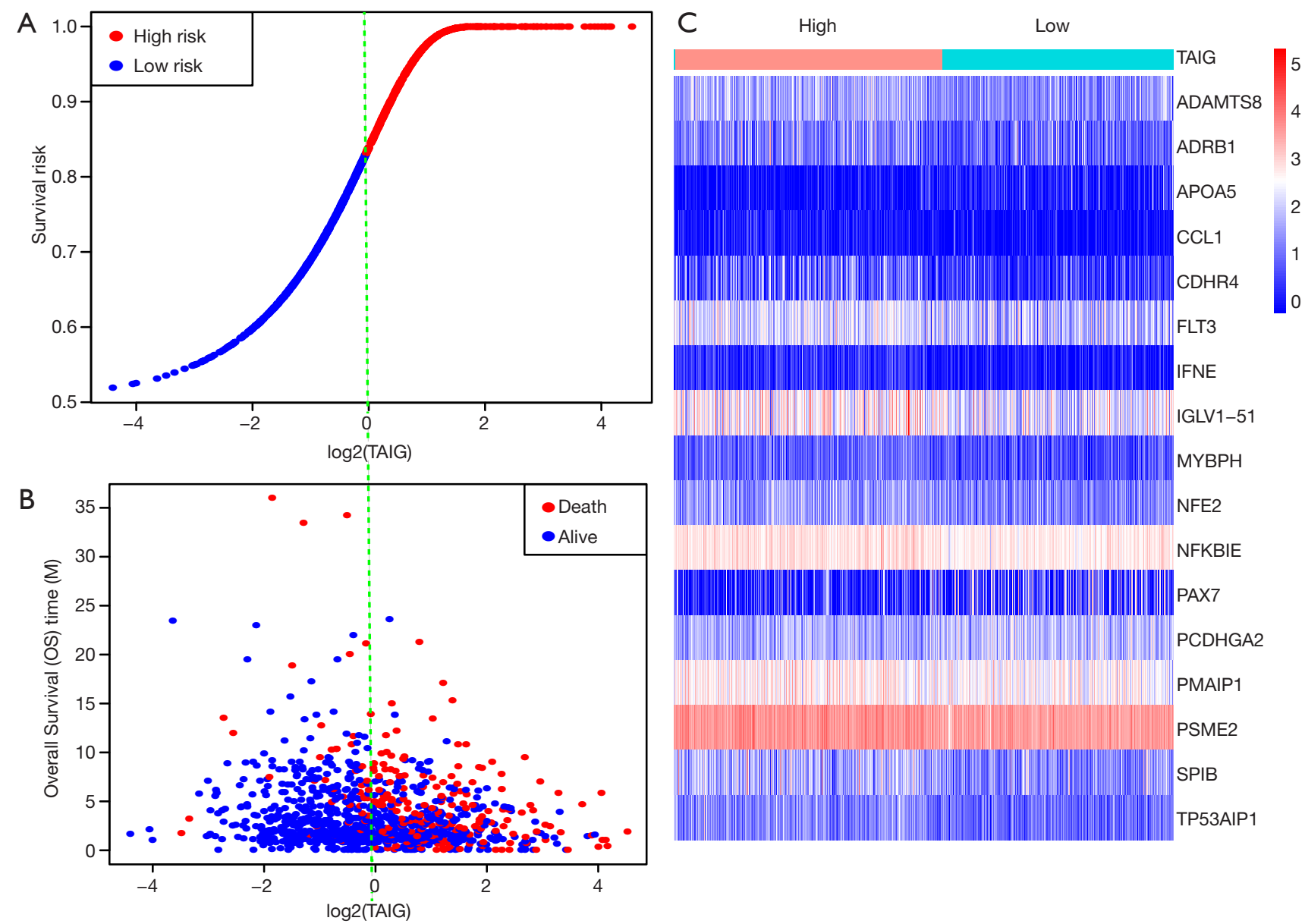

Figure 2 Construction of TAIG and distributions. (A,B) Vital status of patients according to the TAIG levels; (C) the differential levels of identified hub immune signature between two TAIG groups via heatmap plot.

sum of immune fractions in each sample was equal to one (Figure 6).

\section{Differential abundance of tumour-infiltrating immune cells in two TAIG groups}

Since we analysed the potential associations of immune signature with several immune cells, we speculated that there were differential distributions of immune cells in the two TAIG groups (see Figure S1). The heatmap exhibited the infiltrating difference of immune cells in two groups where the colours ranging from green to red represented the infiltration density from low to high (Figure 7). Additionally, we utilized the Wilcoxon rank-sum test to accurately compare the difference and found that several immune cells conferred significantly lower infiltrating density in the high TAIG groups, including $\mathrm{CD}^{+} \mathrm{T}$ cells $(\mathrm{P}=0.031)$, memory resting $\mathrm{CD}^{+}{ }^{+} \mathrm{T}$ cells $(\mathrm{P}=0.026), \mathrm{M} 0$ macrophages $(\mathrm{P}=0.023)$, as well as M2 macrophages $(\mathrm{P}=0.048)$. Several previous studies have already demonstrated that lower immune infiltration in the tumour microenvironment likely results in a poor prognosis in BC. Based on the integrative analysis, we inferred that the risk factor of TAIG was associated with lower immune infiltrates, thus leading to worse survival outcomes.

\section{Discussion}

Although both the DEGs and the immune environment of $\mathrm{BC}$ have been well studied, considering that $\mathrm{BC}$ has the highest incidence/mortality among female malignant tumours types, the immune biomarkers for the prediction of the tumour, especially in the TIL communities, are lacking. Taking into account the importance of immune infiltration 
A

Hazard ratio

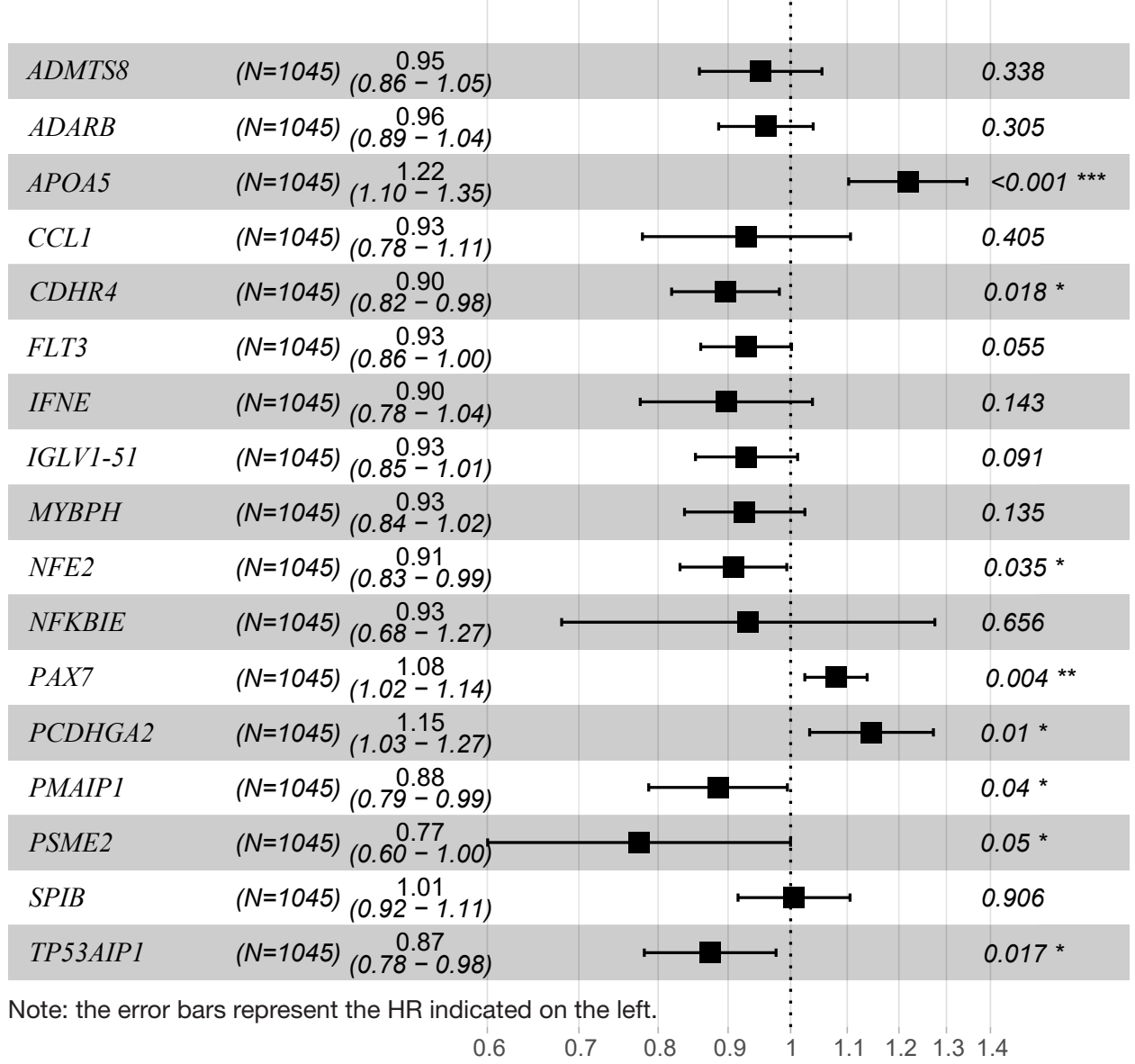
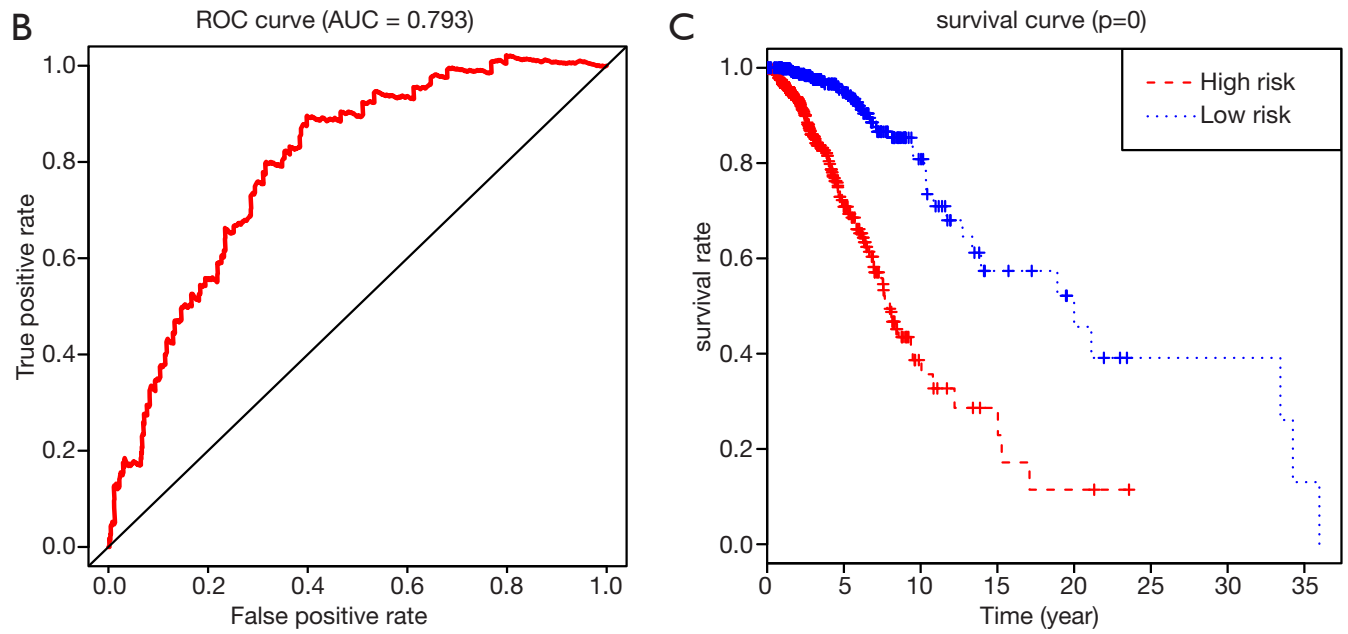

Figure 3 Assessment of TAIG in predicting prognosis of breast cancer. (A) Multivariate Cox results of 17 hub immune signature exhibited by forest plot; (B) the 3-year AUC of ROC curve was 0.793, indicating better predictive power; (C) the patients with high TAIG levels revealed poor survival outcomes in Kaplan-Meier analysis with $\mathrm{P}<0.001$. ${ }^{*}, \mathrm{P}<0.05 ;{ }^{* *}, \mathrm{P}<0.005$; ${ }^{* *}, \mathrm{P}<0.001$. ROC, receiver operating characteristic; AUC, area under the ROC curve. 

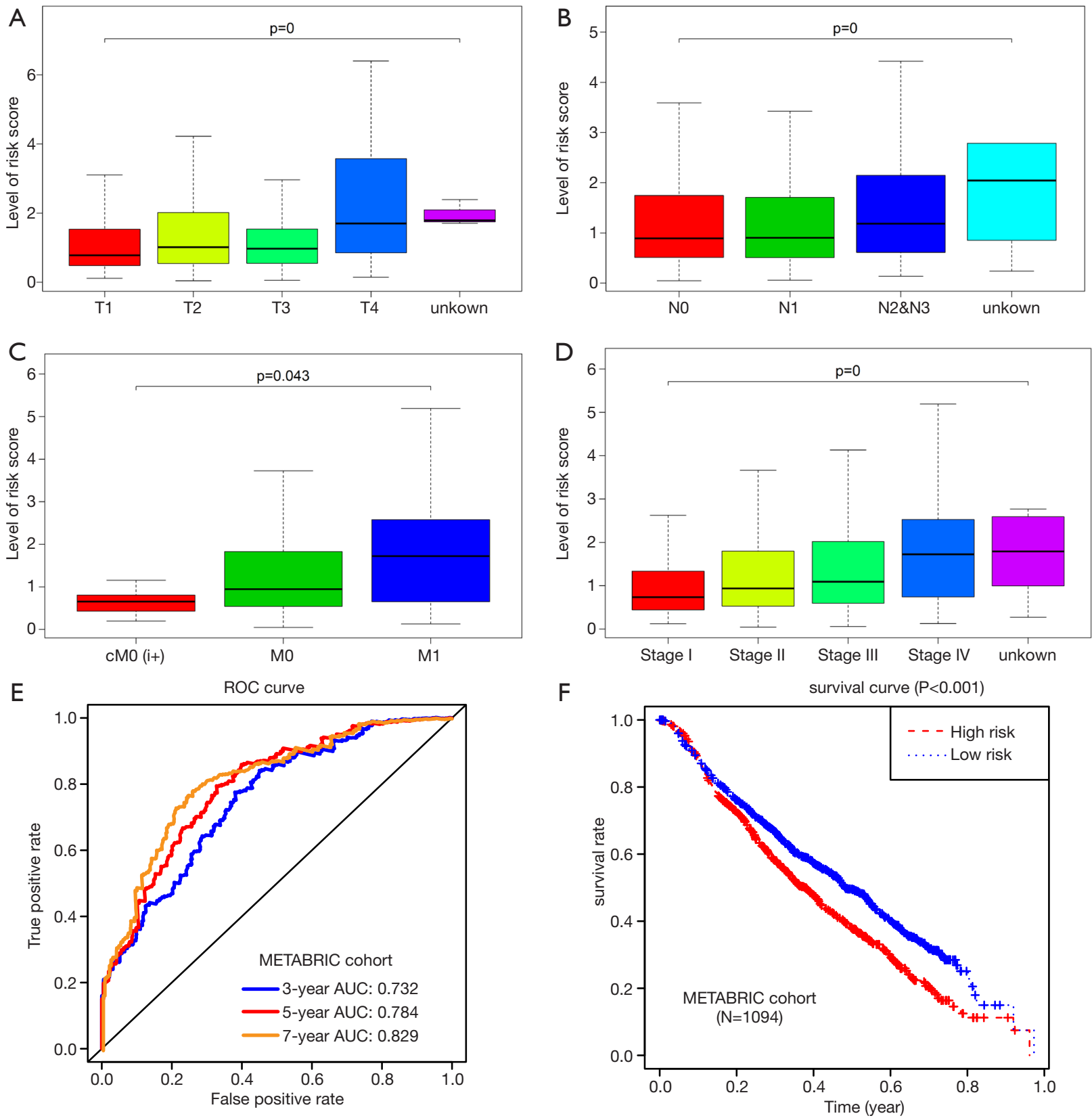

Figure 4 Correlation analysis of TAIG with clinical variables. (A,B,C,D) Kruskal-Wallis test suggested that high TAIG levels correlated with high AJCC-TNM stages and advanced pathological stages $(\mathrm{P}<0.01)$. (E,F) The validation process of TAIG in METABRIC cohort $(\mathrm{N}=1,094)$.

in the development and proliferation of cancer, the finding of novel BC immune-related biomarkers may prove useful for immunotherapy. The present study established a robust immune-related risk signature for BC using the TCGA datasets. With the adjustment of the TAIG risk model, these signatures were able to predict the patients' OS. In addition, we found a significant correlation of these signatures to immune cells according to the TIMER datasets. BC patients with a high immune risk of the selected signatures can have a large deviation in the inflammatory microenvironment. Finally, 17 immune-related signatures were selected as the hub immune genes. According to the KEGG and GO functional results, our signatures take part in cytokine-cytokine receptor interactions, Th1 and Th2 

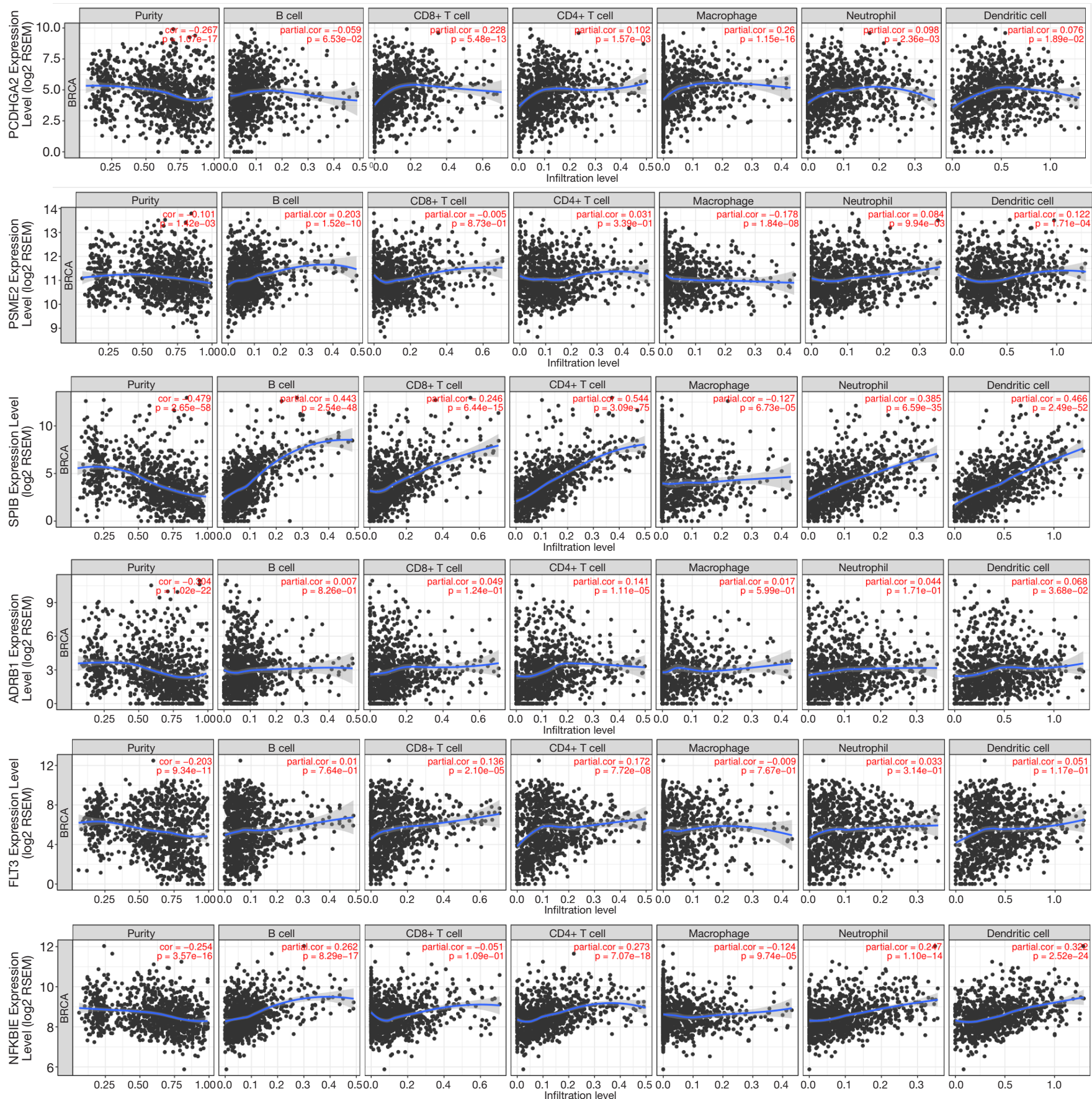

Figure 5 Integrative analysis between hub identified immune signature with tumor-infiltrating immune cells.

cell differentiation and the JAK-STAT signalling pathway. This can further explain the effect of these signatures on the relative cytokines and their receptors in the pathogenesis of cancer (5). Taken together, a higher TAIG levels means a poorer OS. The BC patients with high immune risk levels could stimulate the deterioration of the tumour and accelerate the progression of $\mathrm{BC}$, leading to poor OS.

To determine the clinical values of these signature, the associations among TAIG risk level, AJCC-TNM stages, and patients' OS were evaluated. Patients with a high 


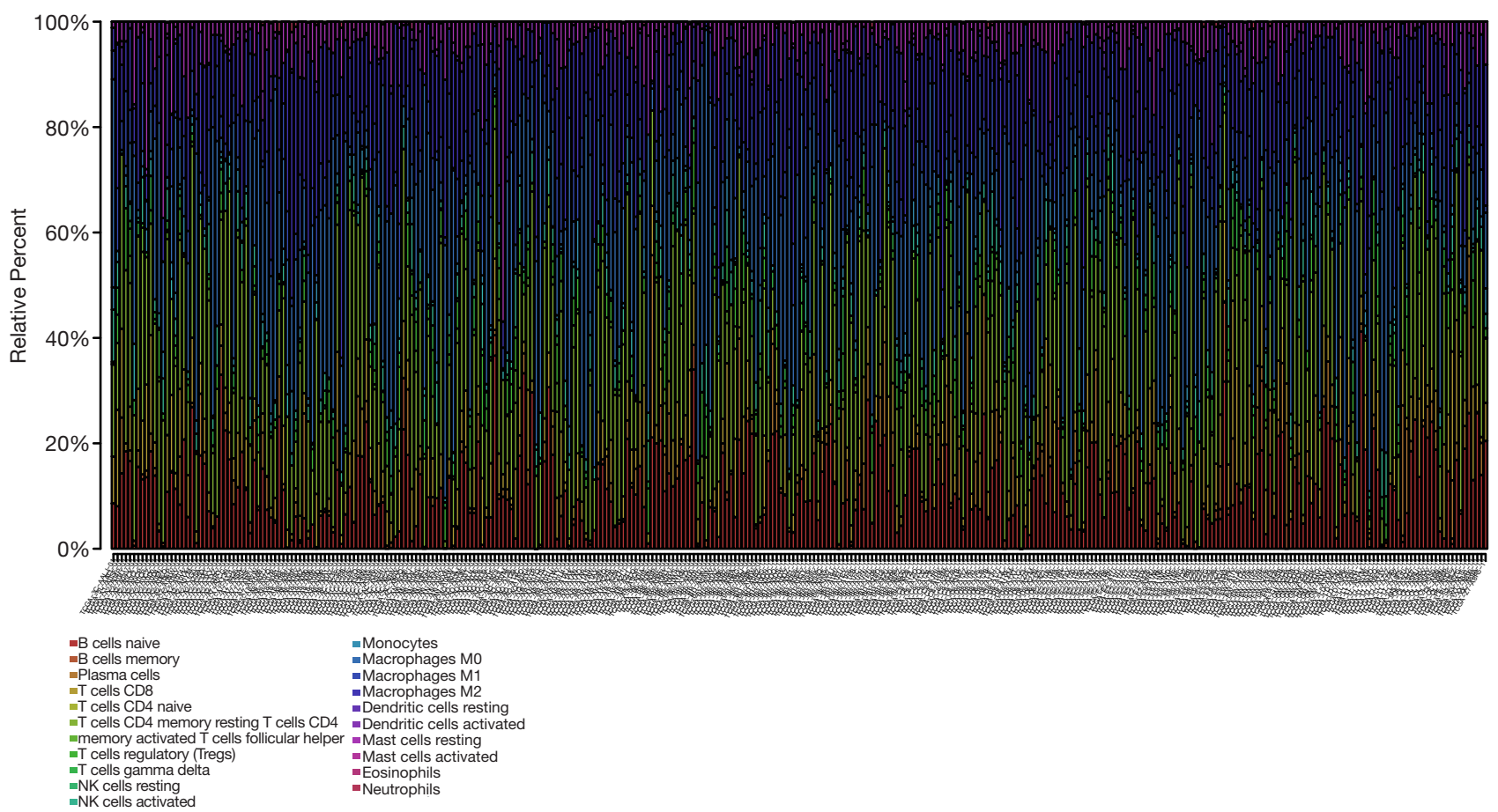

Figure 6 Estimation of fractions of immune cells using the CIBERSORT algorithm, where the 22 immune cells were annotated by various colors under the legend.

immune risk score tended to have high AJCC-TNM stages and advanced pathological stages. Our investigation was the first to uncover the immune-related differences between the clinical stages of BC. Based on the results, the high immune risk microenvironment may promote the development and recurrence of $\mathrm{BC}$, which leads to an advanced stage and relapse of the tumour. Therefore, our signatures can also be regarded as predictors of the development, recurrence and survival outcomes of BC. The multivariate analysis result indicated that the immune-related risk signatures cannot only be independent predictors of $\mathrm{BC}$ in the clinic but also potential immunotherapy targets for BC patients in the future.

According to the results, 17 hub immune signatures with tumour purity, or several important immune cells, and PCDHGA2, SPIB, ADRB1, FLT3, and NFKBIE were the most significant factors. PCDH is expressed in the nervous system mainly, and $\mathrm{PCDH} \gamma$ participates in synaptogenesis and mediates the connection between astrocytes and neurons (13). PCDHGA2 belongs to the PCDH $\gamma$ family and regulates the function of the nervous system, such as synaptic generation (14) and neuronal growth, maturation and differentiation (15), among cells in the intracellular region. PCDHGA2 mutations can be regarded as the signature correlated with several cancer types. In Ping's research (16), high-recurrent-risk patients of invasive lobular cancer of the breast tended to have PCDHGA2 mutations. Song's research demonstrated that (17), in aggressive papillary thyroid microcarcinomas patients, PCDHGA2 can promote the aggressiveness of the tumour. The overexpression of PCDHGA2 mutations could accelerate the metastasis of the tumour by its adhesioninduced function. Therefore, we suggest that PCDHGA2 is a bridge between neuroscience and immune-oncology.

Because of the conserved ETS domains, the ETS family could induce specific DNA binding. SPIB is a transcription factor of the ETS family that is homologous with the SPI1 (PU.1) gene in sequence. In a previous study, SPIB participated in the growth of B lymphocyte and plasmacytoid dendritic cells ( $\mathrm{pDCs}$ ), thus regulating the immune function of these cells (18). Considering the immunomodulatory role of SPIB in B lymphocytes, the copy amplification, translocation and chromosome mutations of SPIB can promote tumour-immune microenvironment remodelling $(19,20)$. There have been studies showing that SPIB regulates the infiltration of blood 

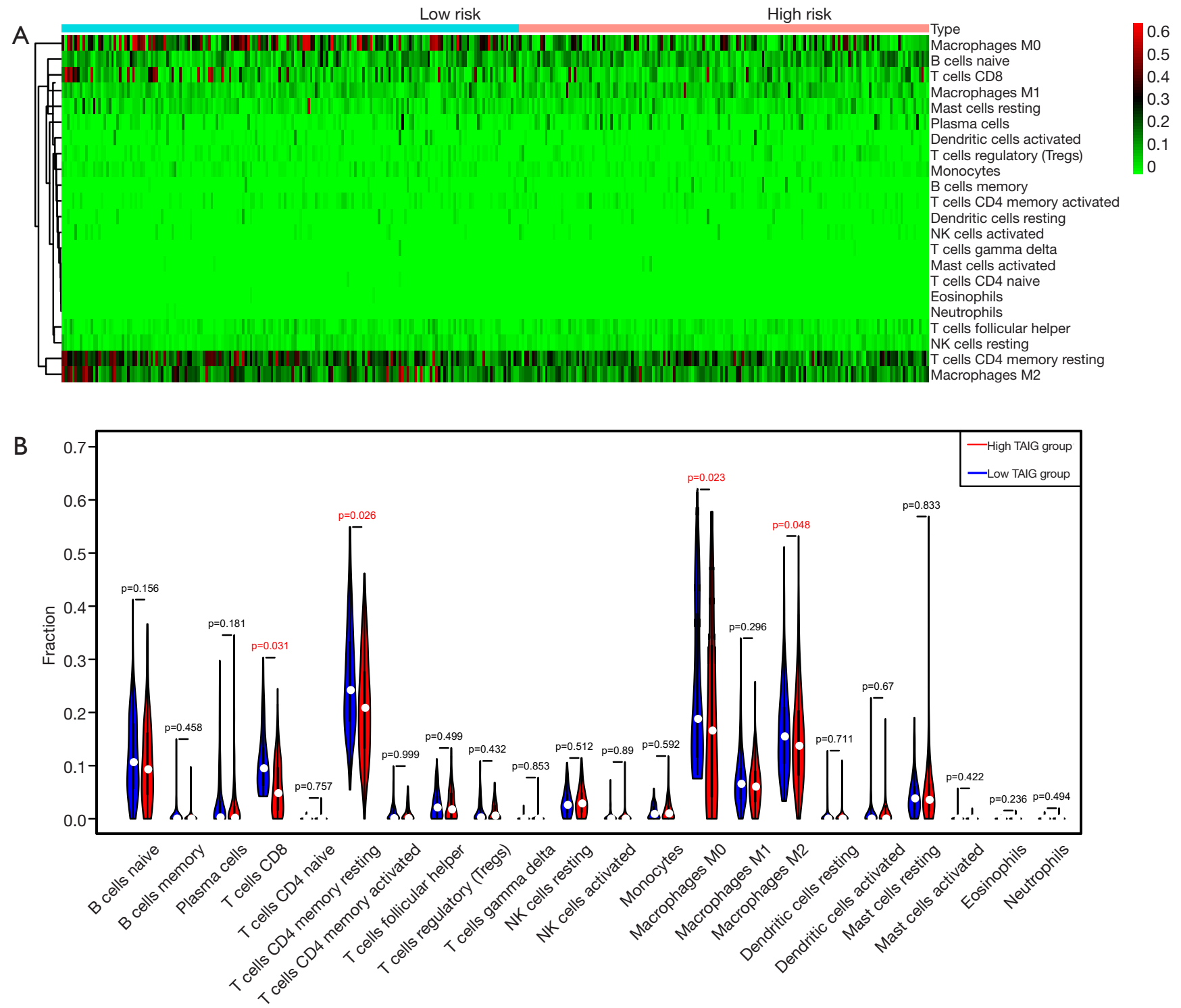

Figure 7 Differential distributions of immune cells in two TAIG groups. (A) The heatmap exhibited the infiltrating difference of immune cells in two groups where the colors ranging from green to red represented the infiltration density of low to high. (B) Wilcoxon ranksum test accurately compared the difference and indicated that several immune cells conferred significantly lower infiltrating density in high TAIG groups, including $\mathrm{CD}^{+} \mathrm{T}$ cell $(\mathrm{P}=0.031)$, memory resting $\mathrm{CD}^{+} \mathrm{T}$ cell $(\mathrm{P}=0.026), \mathrm{M} 0$ macrophage $(\mathrm{P}=0.023)$, as well as $\mathrm{M} 2$ macrophage $(\mathrm{P}=0.048)$.

cells, which was one of the suggested mechanisms for several leukaemias, such as acute lymphoblastic leukaemia (21) and acute myeloid leukaemia (22) Moreover, SPIB is also overexpressed in hepatoma and lung carcinoma tissue (23). Regarding its immunologic function in B-lymphocytes, we suggest that SPIB is widely involved in the invasion and metastasis of cancer.
Similar to SPIB, FLT3 is another important signature in haematological diseases. In myeloid cells, FLT3 is expressed restrictedly in CD34 ${ }^{+}$cells (24). FLT3 and its ligand (FLT3-L), assembled by marrow stroma cells, play an important role in self-renewal, proliferation and differentiation of hematopoietic stem cells. Therefore, FLT3 is a critical factor for leukaemia patients. FLT3 internal 
tandem duplication (FLT3-ITD) mutations are associated with poor survival outcomes of AML (25). Furthermore, in the ALL patients, FLT3 mutations usually led to an increase in lymphoid markers such as CD7, CD13, CD34, CD117 (26). Hence, we consider that FLT3 connected the lymphoblast immunity reaction and the immunological microenvironment. In our study, FLT3 is overexpressed in $\mathrm{CD} 7$ and $\mathrm{CD} 13$ of $\mathrm{BC}$ patients with high immune risk. These findings suggest that tumour microenvironment remodelling caused by immunomodulatory dysfunction can promote the expression of FLT3 in BC patients.

Most prior studies support a dominant role of the NFKB pathway in $\mathrm{BC}$, which was one of the multiple mechanisms of immune regulation to restrain immune responses and regulate their propagation (27). The inhibition of $\mathrm{p} 53$ transcription resulting from NFKB pathway activation could limit the transcription of other apoptosis-promoting genes, thus inducing the proliferation of tumour cells. Hence, the blockade of the NFKB pathway can be regarded as an immune approach in BC immunotherapy (28). As an important inhibitor of the NFKB pathway, NFKBIE were strongly associated with chronic lymphocytic leukaemia (CLL) and melanoma, where NFKBIE mutations occur in $7 \%$ of CLL patients with a poor prognosis (29) and $14.5 \%$ of melanoma patients with a poor prognosis (30). Additionally, NFKBIE regions in granulocytes and macrophages participate in the regulation of the autoimmune response, which was an important immune signature in the rheumatoid arthritis microenvironment (31). These studies show that NFKBIE can limit tumour trans-differentiation from immune infiltration by the NFKB pathway.

Furthermore, we tried to investigate the correlation of these immune signatures and patient prognosis. Further studies showed that immune cells had a significantly lower infiltrating density in high TAIG groups, including $\mathrm{CD}^{+} \mathrm{T}$ cells $(\mathrm{P}=0.031)$, memory resting $\mathrm{CD}^{+} \mathrm{T}$ cells $(\mathrm{P}=0.026), \mathrm{M} 0$ macrophages $(\mathrm{P}=0.023)$, and $\mathrm{M} 2$ macrophages $(\mathrm{P}=0.048)$. This is in agreement with the study of Tawfik (32) and Bense (33): the decrease of immune infiltrating cells in the tumour microenvironment may be related to poor prognosis in BC. In our study, high-TAIG group patients had less $\mathrm{CD}^{+}$and $\mathrm{CD} 4^{+}$ T-cell infiltration, leading to the poor prognosis of the patients. Nevertheless, further investigations are needed to confirm the mechanism of these signatures in the immune microenvironment.

Taken together, our study is the first to identify and validate 17 immune-related genes according to the
TAIG scoring system, and our results can further prove a connection between the TAIG score and the prognosis of $\mathrm{BC}$ patients, which could indicate the immune infiltration intensity in the $\mathrm{BC}$ microenvironment. These selected signatures can also provide novel immune targets for BC immune-related treatment in the future.

Compared with the traditional study of $\mathrm{BC}$ bio-markers, our study analysed many clinical samples in the public database and focused on the expression of immune-related genes in immune cells. With the study of the characteristics of cellular immune infiltration, the relationship between the regulation of immune-related genes and the incidence and prognosis of $\mathrm{BC}$ was investigated in depth. The use of CIBERSORT also greatly improved the efficiency of research and avoided the heavy experimental process. This study used a large number of queues in the TCGA database to identify and validate immune markers and to further assess their clinical risks. However, because this study entirely relies on fitting, its clinical accuracy needs to be further assessed in cohort studies. In addition, the markers obtained in this study need to be combined with clinical studies to determine their functions and provide a basis for future clinical research.

With the method proposed in this study, we can utilize public databases for immune inflammation studies without the need for physical pathways or understanding the potential cell composition of samples. The method proposed in this study enables us to fully examine the immune inflammation patterns of $\mathrm{BC}$ mentioned in previous studies, to assess the specific distribution of immune cells and the changes of corresponding biomarkers, and to discover new biological relationships among them. The method proposed in this study provides a new therapeutic target for clinical treatment. The feasibility of this study is high, provides a feasible method for future BC immune research and can be extended to other cancer research.

\section{Acknowledgments}

Funding: The Project was supported by the Open fund of Key Laboratory of Ministry of Education for TCM Viscera-State Theory and Applications, Liaoning University of Traditional Chinese Medicine. The Project was supported by the Shenyang Science and Technology Project (18-013-0-44). The Project was supported by the Natural Science Fund Project of Science and Technology Department of Liaoning Province (No. 0180550645). The Project was supported by Research Administration Office 
of Liaoning Economy Vocational and Technical College (Ljz2018-qn-07).

\section{Footnote}

Conflicts of Interest: The authors have no conflicts of interest to declare.

Ethical Statement: The authors are accountable for all aspects of the work in ensuring that questions related to the accuracy or integrity of any part of the work are appropriately investigated and resolved.

\section{References}

1. Bray F, Ferlay J, Soerjomataram I, et al. Global cancer statistics 2018: GLOBOCAN estimates of incidence and mortality worldwide for 36 cancers in 185 countries. CA Cancer J Clin 2018;68:394-424.

2. Maishman T, Cutress R, Hernandez A, et al. Local Recurrence and Breast Oncological Surgery in Young Women With Breast Cancer: The POSH Observational Cohort Study. Ann Surg 2017;266:165-72.

3. van Ramshorst MS, van Werkhoven E, Mandjes IAM, et al. Trastuzumab in combination with weekly paclitaxel and carboplatin as neo-adjuvant treatment for HER2positive breast cancer: The TRAIN-study. Eur J Cancer 2017;74:47-54.

4. The Lancet. Breast cancer targeted therapy: successes and challenges. Lancet 2017;389:2350.

5. Inoue K, Ninomiya J, Saito T, et al. Correction to: Eribulin, trastuzumab, and pertuzumab as first-line therapy for patients with HER2-positive metastatic breast cancer: a phase II, multicenter, collaborative, open-label, single-arm clinical trial. Invest New Drugs 2019;37:592-3.

6. Boers-Sonderen MJ, de Geus-Oei LF, Desar IM, et al. Temsirolimus and pegylated liposomal doxorubicin (PLD) combination therapy in breast, endometrial, and ovarian cancer: phase Ib results and prediction of clinical outcome with FDG-PET/CT. Target Oncol 2014;9:339-47.

7. Nahleh Z, Botrus G, Dwivedi A, et al. Bevacizumab in the neoadjuvant treatment of human epidermal growth factor receptor 2-negative breast cancer: A metaanalysis of randomized controlled trials. Mol Clin Oncol 2019;10:357-65.

8. Foukakis T, Lövrot J, Matikas A, et al. Immune gene expression and response to chemotherapy in advanced breast cancer. Br J Cancer 2018;118:480-8.
9. De La Cruz LM, McDonald ES, Mick R, et al. Anti-HER2 CD4(+) T-Helper Type 1 Immune Response is Superior to Breast MRI for Assessing Response to Neoadjuvant Therapy in Patients with HER2-Positive Breast Cancer. Ann Surg Oncol 2017;24:1057-63.

10. Cimino-Mathews A, Thompson E, Taube JM, et al. PD-L1 (B7-H1) expression and the immune tumor microenvironment in primary and metastatic breast carcinomas. Hum Pathol 2016;47:52-63.

11. Emens LA. Breast cancer immunobiology driving immunotherapy: vaccines and immune checkpoint blockade. Expert Rev Anticancer Ther 2012;12:1597-611.

12. Egelston CA, Avalos C, Tu TY, et al. Human breast tumor-infiltrating CD8(+) T cells retain polyfunctionality despite PD-1 expression. Nat Commun 2018;9:4297.

13. Thu CA, Chen WV, Rubinstein R, et al. Singlecell identity generated by combinatorial homophilic interactions between alpha, beta, and gamma protocadherins. Cell 2014;158:1045-59.

14. Garrett AM, Weiner JA. Control of CNS synapse development by \{gamma\}-protocadherin-mediated astrocyte-neuron contact. J Neurosci 2009;29:11723-31.

15. Frank M, Ebert M, Shan W, et al. Differential expression of individual gamma-protocadherins during mouse brain development. Mol Cell Neurosci 2005;29:603-16.

16. Ping Z, Siegal GP, Harada S, et al. ERBB2 mutation is associated with a worse prognosis in patients with $\mathrm{CDH} 1$ altered invasive lobular cancer of the breast. Oncotarget 2016;7:80655-63.

17. Song J, Wu S, Xia X, et al. Cell adhesion-related gene somatic mutations are enriched in aggressive papillary thyroid microcarcinomas. J Transl Med 2018;16:269.

18. Willis SN, Tellier J, Liao Y, et al. Environmental sensing by mature $\mathrm{B}$ cells is controlled by the transcription factors PU.1 and SpiB. Nat Commun 2017;8:1426.

19. Takagi $Y$, Shimada K, Shimada S, et al. SPIB is a novel prognostic factor in diffuse large B-cell lymphoma that mediates apoptosis via the PI3K-AKT pathway. Cancer Sci 2016;107:1270-80.

20. Yang Y, Shaffer AL 3rd, Emre NC, et al. Exploiting synthetic lethality for the therapy of ABC diffuse large B cell lymphoma. Cancer Cell 2012;21:723-37.

21. Sokalski KM, Li SK, Welch I, et al. Deletion of genes encoding PU.1 and Spi-B in B cells impairs differentiation and induces pre-B cell acute lymphoblastic leukemia. Blood 2011;118:2801-8.

22. Mullighan CG, Goorha S, Radtke I, et al. Genome-wide analysis of genetic alterations in acute lymphoblastic 
leukaemia. Nature 2007;446:758-64.

23. Ho YJ, Lin YM, Huang YC, et al. Tissue microarraybased study of hepatocellular carcinoma validating SPIB as potential clinical prognostic marker. Acta Histochem 2016;118:38-45.

24. Rappold I, Ziegler BL, Kohler I, et al. Functional and phenotypic characterization of cord blood and bone marrow subsets expressing FLT3 (CD135) receptor tyrosine kinase. Blood 1997;90:111-25.

25. Fischer M, Schnetzke U, Spies-Weisshart B, et al. Impact of FLT3-ITD diversity on response to induction chemotherapy in patients with acute myeloid leukemia. Haematologica 2017;102:e129-31.

26. Hoehn D, Medeiros LJ, Chen SS, et al. CD117 expression is a sensitive but nonspecific predictor of FLT3 mutation in T acute lymphoblastic leukemia and T/myeloid acute leukemia. Am J Clin Pathol 2012;137:213-9.

27. Jiao X, Wood LD, Lindman M, et al. Somatic mutations in the Notch, NF-KB, PIK3CA, and Hedgehog pathways in human breast cancers. Genes Chromosomes Cancer 2012;51:480-9.

28. Ling J, Kumar R. Crosstalk between NFkB and glucocorticoid signaling: a potential target of breast cancer therapy. Cancer Lett 2012;322:119-26.

29. Tuveson D, Rai KR. Augmenting NF-kappaB in poor-risk CLL: A general paradigm for other cancers? J Exp Med 2015;212:830-1.

30. Shain AH, Garrido M, Botton T, et al. Exome sequencing of desmoplastic melanoma identifies recurrent NFKBIE promoter mutations and diverse activating mutations in the MAPK pathway. Nat Genet 2015;47:1194-9.

31. Imamura H, Yoshina S, Ikari K, et al. Impaired NFKBIE gene function decreases cellular uptake of methotrexate by down-regulating SLC19A1 expression in a human rheumatoid arthritis cell line. Mod Rheumatol 2016;26:507-16.

32. Tawfik O, Kimler BF, Karnik T, et al. Clinicopathological correlation of PD-L1 expression in primary and metastatic breast cancer and infiltrating immune cells. Hum Pathol 2018;80:170-8.

33. Bense RD, Sotiriou C, Piccart-Gebhart MJ, et al. Relevance of Tumor-Infiltrating Immune Cell Composition and Functionality for Disease Outcome in Breast Cancer. J Natl Cancer Inst 2016. doi: 10.1093/jnci/ djw192
Cite this article as: Zhang B, Wang Q, Fu C, Jiang C, Ma S. Exploration of the immune-related signature and immune infiltration analysis for breast ductal and lobular carcinoma. Ann Transl Med 2019;7(23):730. doi: 10.21037/atm.2019.11.117 


\section{Supplementary}

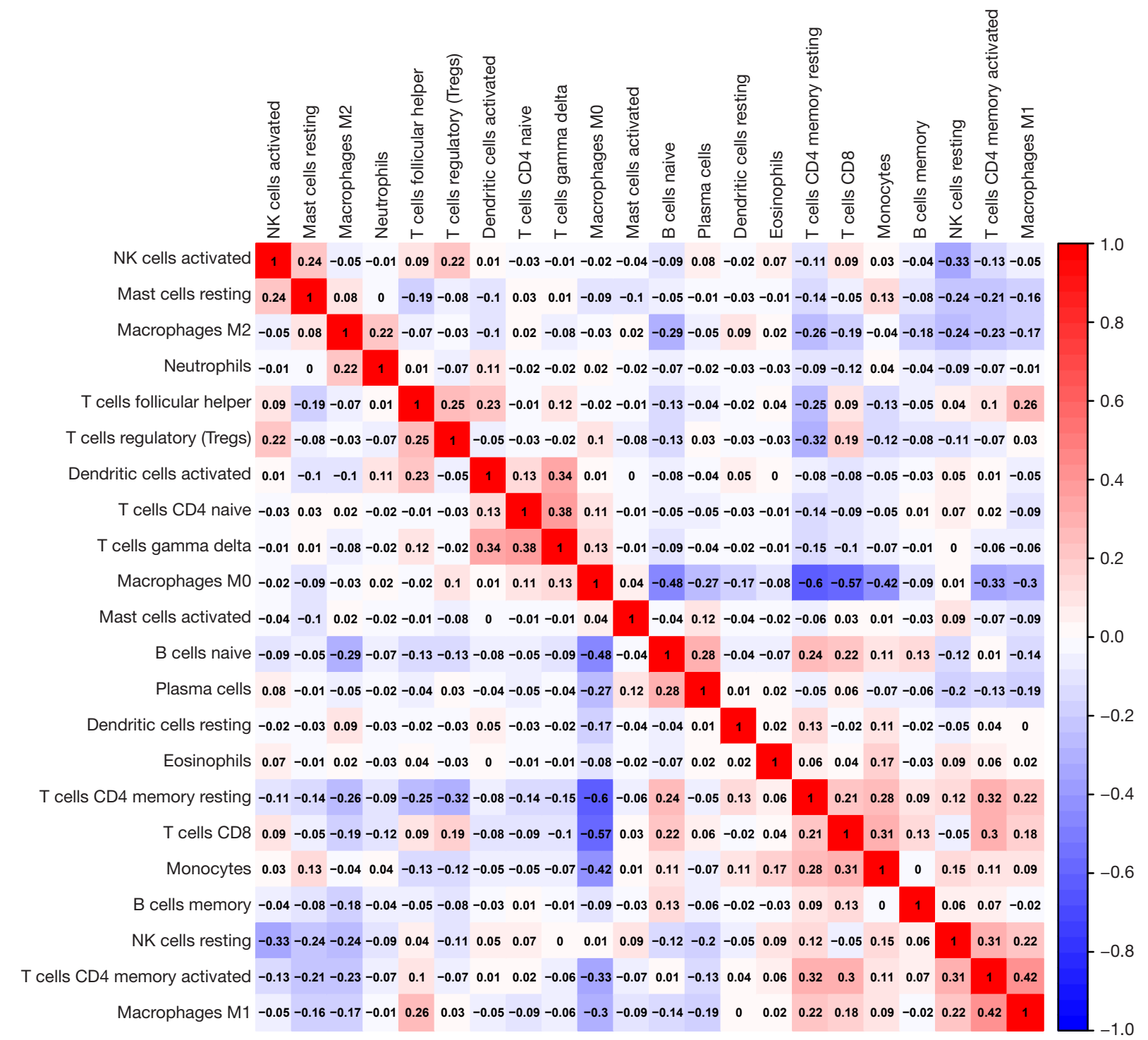

Figure S1 Correlation between tumor-infiltrating immune cells where red represented the positive correlation while blue represented the negative correlation. 